\title{
Association of tau accumulation and atrophy in mild cognitive impairment: a longitudinal study
}

Gang Xu

Anhui Polytechnic University

Shuzhan Zheng

Anhui Polytechnic University

Zhilong Zhu

Anhui Polytechnic University

Xiaofeng Yu

Anhui Polytechnic University

Jian Jiang

Wannan Medical College

Juanjuan Jiang ( $\square$ jiangjuanjuan@ahpu.edu.cn )

Anhui Polytechnic University https://orcid.org/0000-0003-1875-4807

Zhaohu Chu

Wannan Medical College

Original research

Keywords: tau, flortaucipir, atrophy, mild cognitive impairment, longitudinal change

Posted Date: March 26th, 2020

DOI: https://doi.org/10.21203/rs.3.rs-19015/v1

License: (1) (1) This work is licensed under a Creative Commons Attribution 4.0 International License.

Read Full License

Version of Record: A version of this preprint was published at Annals of Nuclear Medicine on August 12th, 2020. See the published version at https://doi.org/10.1007/s12149-020-01506-2. 


\section{Abstract}

Background To examine the patterns of longitudinal tau accumulation and cortical atrophy and their association in patients with mild cognitive impairment (MCl).

Methods We collected 23 participants (60-89 years old, 11 male/12 female) with $\mathrm{MCl}$ from the Alzheimer's Disease Neuroimaging Initiative database. All participants underwent $18 \mathrm{~F}$ flortauirpir (FTP) positron emission tomography (PET) and structural magnetic resonance imaging (MRI) scans at the baseline and follow-up visits (12-36 months). General linear models with covariates (baseline age, sex) were used to detect brain areas of significant tau accumulation and atrophy over time. The mediation analysis was employed to explore the potential reason for sequential biomarker changes in $\mathrm{MCl}$ progression, adjusting for baseline age, sex, education.

Results Voxelwise tau accumulation in $\mathrm{MCl}$ patients was predominantly located in inferior temporal, middle temporal, parietal cortex, posterior cingulate, precuneus as well as temporo-parietal regions $(\mathrm{P}<$ 0.001 ), and MRI atrophy included inferior-middle temporal, parietal lobe, cerebellum and precuneus $(P<$ $0.001)$. Longitudinal FTP accumulation was moderately associated with MRI cortical atrophy $(r=0.409$, $95 \% \mathrm{Cl}: 0.405-0.414, \mathrm{P}<0.01)$. Regional analyses indicated significant bivariate associations between MRI cortical atrophy and FTP accumulation (baseline FTP cortical uptake and longitudinal FTP change). The result of the mediation analysis showed the relationship between baseline FTP uptake and longitudinal cortical atrophy was partly mediated by the longitudinal FTP cortical change (indirect effect: $0.0107, \mathrm{P}=0.04)$.

Conclusions Our finding provides a preliminary description of the patterns of longitudinal FTP accumulation and cortical atrophy in $\mathrm{MCl}$ progression, and $\mathrm{MCl}$ patients with high tau binding level show increase risk of longitudinal tau accumulation, atrophy and cognitive decline.

\section{Introduction}

Alzheimer's disease (AD) is a quite complex progressive cognitive disease leading to dementia, manifesting in functional alterations and structural atrophy throughout the brain [1]. AD is typically described in focal terms: it is characterized pathologically by extracellular accumulation of amyloid- $\beta$ plaques and by intracellular accumulation of neurofibrillary tangles composed of hyperphosphorylated tau [2]. Mild cognitive impairment $(\mathrm{MCl})$ due to $A D$ is the intermediate pathological stage between healthy elders and $A D$, individual with $\mathrm{MCl}$ are at increased risk of progressing $A D$. The proposed downstream consequence of developing pathology can be visualized and quantified in living patients with positron emission tomography (PET) with various radiotracers and structural MRI [3]. Those observing pathophysiological events in the brain of clinical MCI patients is critically important to develop the understanding of the neurodegenerative cascade progressing to $A D$ and the relationship between protein accumulation, cortical atrophy and cognitive impairment. The development of neurological trajectories can help clinical prevention to halt the progression of pathology and delay cognitive decline. 
Based on postmortem studies, the cognitive deficits correlates for tau pathology are generally stronger than for amyloid- $\beta$ pathology [4-6]. Converging evidence demonstrates that the development of amyloid and tau pathology may begin independently, but that amyloid- $\beta$ plaques accumulate decades before cognitive symptoms and precedes and accelerates the spread of tau, which further precipitate cognitive deficits $[5,7,8]$. More recently, neuroimaging studies have suggested that intimate associations between the spatial pattern of tau accumulation and neuropathological alterations (i.e., cortical atrophy, glucose hypometabolism), and those neurodegeneration patterns likely interact over time [9-12]. The spatial pattern of tau may have prominently intervened in the progression of $\mathrm{MCl}$ and preclinical $A D$. Recent studies about longitudinal neurological disease suggest that tau accumulation is useful to track the disease progression and tau pathology is a major driver of subsequence severe cortical atrophy $[13,14]$. There are few studies that jointly explore the longitudinal tau accumulation and other related cortical atrophy. Note that previous study disclosed the longitudinal tau accumulations and the relationships of tau change to cortical atrophy for healthy elders and patients with $A D$, but missing patients with $\mathrm{MCI}$ [15]. Importantly, the pathophysiological process of $\mathrm{MCl}$ stage is critical to guide clinical management and prevention trials.

Identifying the association between serial tau accumulation and cortical atrophy with concurrent and subsequent may address the gap in our understanding of $\mathrm{MCl}$ progression. In this present study, we prospectively evaluated this association using ${ }^{18} \mathrm{~F}$ flortaucipir (FTP) PET and structural MRI images in a group of patients with $\mathrm{MCl}$. To that end, this study aimed to assess the patterns of longitudinal tau change and MRI atrophy, the spatial correlation between tau accumulation and cortical atrophy, as well as the potential reason for sequential biomarker changes in $\mathrm{MCl}$ progression.

\section{Materials And Methods Participants}

Data in this study were obtained from the Alzheimer's Disease Neuroimaging Initiative (ADNI) database (adni.loni.usc.edu). The ADNI is a longitudinal and cross-sectional multicenter study that provides a centralized resource for clinical, brain imaging, and fluid biomarker data. The ADNI was launched in 2003 as a public-private partnership, led by Principal Investigator Michael W. Weiner, MD. The primary goal of ADNI has been to test whether serial MRI, PET, other biological markers, and clinical and neuropsychological assessment can be combined to measure the progression of $\mathrm{MCl}$ and early $\mathrm{AD}$.

In this study, we analyzed 23 longitudinal $\mathrm{MCl}$ patients who had undergone baseline tau-PET imaging with FTP and 1.5/3 T structural MRI (sMRI), as well as follow-up FTP and sMRI imaging from January 1 , 2014 to February 13, 2020 (Figure S1). Additional inclusion criteria included age between 55-90 years, a follow-up assessment at least 9 months after the first visit, education of at least 6-grade levels, no serious medical, neurological, or psychiatric condition (Clinical Dementia Rating sum of boxes, CDRSB > 5; MiniMental State Examination, MMSE > 15). All MCl patients were clinically diagnosed by multidisciplinary experts of ADNI according to National Institute of Aging and Alzheimer's Association clinical criteria 
(detailed description in http://www.adni.loni.usc.edu/methods/). Each MCl patients underwent neuropsychological assessments at baseline and follow-up including global cognitive (CDRSB, MMSE) and specific cognitive tasks (memory, executive ability, language, and attention). Clinical characteristics and demographics of all participants were summarized in Table 1. This study was approved by the institutional review board of the $A D N I$, written informed consent was obtained from all participants.

Table 1

All participant characteristics.

\begin{tabular}{|ll|}
\hline Characteristic & Value \\
\hline $\mathrm{n}$ & 23 \\
\hline Age (years) & $73.8 \pm 8.4[60-89]$ \\
\hline Sex $(\mathrm{M} / \mathrm{F}), \mathrm{n}$ & $11(47.8 \%) / 12(52.2 \%)$ \\
\hline Education (year) & $15.7 \pm 2.7[12-20]$ \\
\hline APOE4 alleles: $\mathrm{n}_{0} / \mathrm{n}_{1} / \mathrm{n}_{2} / \mathrm{n}_{\text {missing }}$ & $6 / 11 / 5(1)$ \\
\hline MMSE at baseline & $26.9 \pm 2.1[19-30]$ \\
\hline CDRSB at baseline & $1.47 \pm 1.02[0.5-4.5]$ \\
\hline Baseline to follow-up time (months) & $14.7 \pm 5.6[12-36]$ \\
\hline Note: For continuous variables, mean \pm SD, range (min-max) is indicated. \\
\hline
\end{tabular}

\section{Image acquisition}

The FTP-PET and SMRI image acquisitions were co-collected at the baseline and follow-up visits. For flortaucipir (as known as AV1451 or T807) PET imaging, participants underwent intravenous infusion with $\sim 370 \mathrm{MBq}(10 \mathrm{mCi})$ of flortaucipir $\left({ }^{18} \mathrm{~F}\right)$ and followed $\sim 80$ minutes postinjection later by a continuous brain PET scan acquired six 5-minute frames. FTP-PET scans were reconstructed using an ordered subset expectation maximization (OSEM) algorithm with weighted attenuation and smoothed with a 4-mm Gaussian kernel with scatter correction. T1-weighted sMRI images were also scanned on multiple 1.5/3T MRI scanners using scanner-specific sagittal 3D Magnetization Prepared - RApid Gradient Echo (MPRAGE) sequences. Original PET and sMRI images in ADNI underwent standardized image preprocessing correction protocols to increase signal uniformity across the multicenter instruments. More detailed descriptions about PET and SMRI image acquisitions can be obtained on ADNI database (http://adni.loni.usc.edu/methods/documents/).

\section{Image processing}

The PET and SMRI image preprocess were performed through SPM12 (Statistical Parametric Mapping 12; Wellcome Department of Imaging Neuroscience, Institute of Neurology, London, UK). We designed a longitudinal process pipeline to discover the voxelwise change over time in both PET and MRI images. 
The baseline and follow-up sMRI images were processed via SPM12 pairwise longitudinal registration across individuals [16], obtaining a within-subject midpoint average MRI image and a 3-dimensional Jacobian rate map which indicated an annualized measure of volumetric contraction or expansion as described elsewhere $[14,15]$. The original Jacobian maps were reversed (i.e., multiplied by -1 ) so that higher positive values representing greater annualized MRI atrophy, whereas negative values representing lower annualized MRI expansion [14]. The midpoint MRI images were then segmented into probabilistic gray matter (GM), white matter (WM) and CSF maps via SPM12. The brain tissue segments were processed for further analysis, in which GM segment from each participant's midpoint MRI was masked to exclude basal ganglia and cerebellum and derive a subject-specific cerebral cortical mask, and WM segment was thresholded at 0.95 and then eroded by 1 voxel to derive a subject-specific WM mask.

The FTP-PET frames were realigned and averaged to generate a PET image. The baseline and follow-up PET images were registered into the midpoint MRI space to be aligned with the reversed Jacobian rate map. The subject-specific WM mask from midpoint MRI was used as the reference region to normalized the baseline and follow-up FTP images because this region provided more stable estimates of FTP change $[17,18]$. Partial volume effect (PVE) correction based on Muller-Gartner's algorithm was implemented using SPM's PETPVE12 toolbox $[19,20]$. The FTP change image of each participant was derived by subtracting the baseline FTP image from the follow-up image for each voxel. The reversed Jacobian rate map, the baseline and follow-up FTP images and FTP change image were warped to Montreal Neurological Institute space using the deformation parameters derived from the midpoint MRI segment. The PET images were then smoothed with 6-mm Gaussian kernel, and the reversed Jacobian rate maps used an 8-mm Gaussian kernel. Individual annualized percentage change (APC) image of FTP change was also calculated for each voxel.

For template-warped FTP standardized uptake value ratio (SUVR) images, flortaucipir retention of the region of interest (ROI) was surrogated for each participant by SUVR value. The reversed Jacobian rate, SUVR values of FTP change and APC values were calculated in 5 priori ROls including inferior temporal cortex, precuneus, olfactory; inferior frontal gyrus and cerebral cortex $[13,15]$. The baseline FTP SUVR value also calculated to investigate the association between $\mathrm{MCl}$ progression and it. All FTP and MRI image processing was performed blind to patient clinical status.

\section{Statistical analyses}

Demographic and clinical characteristics at the baseline were summarized with descriptive statistics across all participants (mean, standard deviation, for continuous variables; frequency and percentages for categorical information). For FTP change images, a general linear model (GLM) with covariates (baseline age, sex) was used to detect brain areas of significant tau accumulation over time (i.e., SPM's one-sample t test). The average APC was calculated for these tau significantly changed voxels. Similarly, the reversed Jacobian rate maps also underwent a voxelwise one-sample $t$ test via GLM to reveal regions of significant atrophy over time (-Jacobians $>0$ ). The FTP change images and reversed Jacobian rate maps were used to assess spatial correlations between FTP accumulation and MRI atrophy via pearson correlation. All t test results were presented using an uncorrected voxel threshold of $\mathrm{P}<0.001$ (one-sided). 
The baseline SUVR values, FTP change values and reversed Jacobian rate values of targeted ROI were entered pearson correlation models to measure the associations between pair variables, respectively. To further explore the potential reason for sequential biomarker changes in $\mathrm{MCl}$ progression, all possible indirect pathway between baseline FTP, FTP change and MRI atrophy were tested via mediation analyses, adjusting for baseline age, sex, education. Direct, and indirect associations were tested using a 500iteration bootstrapping procedure. The relationships between cognitive ability change and FTP change were assessed using simple correlation analyses. BrainNet Viewer was employed to show the imaging results [21]. All statistical analyses were implemented using MATLAB 2016b (MathWorks Inc.) and R version 3.61 (https://www.r-project.org/) employing the mediation package [22].

\section{Results}

\section{Characteristics and description of the participants}

The clinical characteristics of the $23 \mathrm{MCl}$ participants eligible for longitudinal analysis were summarized in Table 1. All participants underwent PET and MRI scans at the baseline and follow-up visits (mean time: 14.7 months; range: $12-16$ months). $\mathrm{MCl}$ patients were between 60 and 89 years old at the baseline visit (mean age: $73.8 ; 52.2 \%$ female). In this cohort, $72.7 \%$ of $\mathrm{MCl}$ patients were carried APOE4 alleles $(\mathrm{n}=22$, one is missing). Mean scores on the MMSE (26.7, range: 19-30) and CDRSB (1.47, range: $0.5-4.5)$ were comparable to values previously reported in $\mathrm{MCl}$ patients [23].

\section{Voxelwise longitudinal $\mathrm{MCl}$ pattern}

Figure 1 presented the pattern of longitudinal voxelwise FTP change over time as a significant map based on a one-sample t test performed on the 23 FTP change images. FTP accumulation was predominantly located in inferior temporal, middle temporal, parietal cortex, posterior cingulate, precuneus as well as temporo-parietal regions $(P<0.001$, voxel cluster $>100)$. No significant FTP decrease was detected in elsewhere region. Through FTP significant increase masks overlaid the average APC image, this analysis showed that significant FTP annualized accumulations of $6-11 \%$ in $\mathrm{MCl}$ patients.

To investigate the pattern of $\mathrm{MRI}$ atrophy over time for $\mathrm{MCl}$ patients, we performed a longitudinal voxelbased morphometry (VBM) analysis using reversed Jacobian rate images. As shown in Fig. 2B, MRI atrophy was predominantly involved inferior-middle temporal, parietal lobe, frontotemporal cortex, cerebellum, precuneus as well as parahippocampal (one-sample $t$ test; $P<0.001$, voxel cluster $>100$ ). There was partly overlap between the regions with significant MRI and the regions of significant FTP accumulations (Fig. 2A-B). To explore whether FTP change was independently or synergistically associated with MRI atrophy, a linear model measured the correlation strength using voxelwise statistical maps for FTP change and MRI atrophy (t-statistic, T values). Longitudinal FTP accumulation was moderately associated with MRI cortical atrophy $(r=0.409,95 \%$ confidence interval $(\mathrm{Cl}): 0.405-0.414, \mathrm{P}<$ 0.01 ). These findings revealed that the FTP accumulation and atrophy have synergistically emerged in the progression of $\mathrm{MCl}$ patients. 


\section{ROI analysis}

We used regional SUVR as the surrogate to investigate the baseline FTP accumulations, longitudinal FTP change and atrophy in $\mathrm{MCl}$ progression. Table 2 showed that the inferior temporal cortex had a higher baseline FTP uptake (mean: 1.19) for $\mathrm{MCl}$ patients. $\mathrm{MCl}$ patients showed significant FTP annualized changes across all prior brain regions (all $\mathrm{P}<0.001$ ). In addition, precuneus had the highest annualized difference (APC, $8.54 \%$ per year, $\mathrm{P}<0.001)$ rather than other regions, and the result of $M R I$ atrophy was similar $(0.019$ per year, $\mathrm{P}<0.001)$ when reversed Jacobian rate as the change parameter.

Table 2

Regional longitudinal FTP change and MRI atrophy in $\mathrm{MCl}$ patients.

\begin{tabular}{|llllll}
\hline Region & Baseline FTP SUVR & FTP change & \multicolumn{3}{c}{ MRI atrophy } \\
\cline { 3 - 5 } & & APC & P value & -Jacobian & P value \\
\hline ITC & $1.19 \pm 0.28$ & $8.54 \pm 3.16$ & $<0.001^{\mathrm{a}}$ & $0.019 \pm 0.003$ & $<0.001^{\mathrm{a}}$ \\
\hline PCUN & $1.01 \pm 0.20$ & $9.65 \pm 4.49$ & $<0.001^{\mathrm{a}}$ & $0.015 \pm 0.004$ & $0.003^{\mathrm{a}}$ \\
\hline OLF & $0.97 \pm 0.09$ & $6.87 \pm 3.08$ & $<0.001^{\mathrm{a}}$ & $0.018 \pm 0.007$ & $0.017^{\mathrm{a}}$ \\
IFG & $0.99 \pm 0.17$ & $7.25 \pm 2.91$ & $<0.001^{\mathrm{a}}$ & $0.014 \pm 0.003$ & $<0.001^{\mathrm{a}}$ \\
CC & $1.07 \pm 0.13$ & $5.77 \pm 3.49$ & $<0.001^{\mathrm{a}}$ & $0.005 \pm 0.001$ & $<0.001^{\mathrm{a}}$ \\
\hline
\end{tabular}

Note: Mean \pm standard deviation is given. ${ }^{\text {a }}$. Probability values indicating significant difference at $\mathrm{P}<$ 0.05 (1-sided one sample t test). APC: annualized percentage change (\%); ITC: inferior temporal cortex; PCUN: precuneus; OLF: olfactory; IFG: inferior frontal gyrus; CC: cerebral cortex.

Figure 1. The longitudinal FTP increase in $\mathrm{MCl}$ patients. The left panel was the result's region of onesample t test where $\mathrm{MCl}$ patients showed a significant FTP increase over time (voxel $\mathrm{P}<0.001$, uncorrected). The right panel showed that voxelwise average annualized percentage change (APC) in significant FTP change region.

Figure 2. The spatial statistic maps of FTP accumulation and MRI atrophy and cross-modal correlation. (A) The significant FTP change statistic map derived from FTP change images of each $\mathrm{MCl}$ patient $(n=23$, one-sample t test, $P<0.001)$. (B) The significant MRI atrophy statistic map derived from reversed Jacobian rate map of each patient $(n=23$, one-sample $t$ test, $P<0.001)$. (C) The crossmodal correlation between FTP accumulation and MRI atrophy derived from voxelwise statistic maps ( $T$ value). Correlation was assessed on all voxels of the cerebral cortex.

Figure 3. Bivariate associations between baseline FTP and subsequent longitudinal FTP change/MRI atrophy across the $23 \mathrm{MCl}$ patients. For $95 \%$ confidence interval $(95 \% \mathrm{Cl})$, the bootstrapping with 1000 permutations was performed.

Figure 4. Diagram of mediation model pathway relating baseline FTP, longitudinal FTP change and MRI atrophy. Altogether, the pathways explain $96.9 \%$ of the direct effect. Mediation analysis showed that longitudinal FTP change mediated the effect of baseline FTP on longitudinal MRI atrophy. 
Results from voxelwise analysis denoted that the MRI cortical atrophy was associated with FTP accumulation in $\mathrm{MCl}$ progression. We next explored the relationships between baseline FTP and subsequent longitudinal alterations (FTP change and MRI atrophy) across patients, using cerebral cortex's measurements. Figure 3 showed the bivariate association between MRI cortical atrophy (mean reversed Jacobian rate in cerebral cortex) and FTP accumulation (baseline FTP cortical SUVR and longitudinal FTP change (SUVR/time)). There was a strong correlation between baseline FTP and subsequent annualized FTP increase $(r=0.71,95 \% \mathrm{Cl}$ : 0.43-0.78, $\mathrm{P}<0.001)$, compared with moderate correlations with MRI atrophy $(r=0.41,95 \% \mathrm{Cl}: 0.10-0.61, \mathrm{P}<0.001)$. And longitudinal FTP change was significant associated with MRI atrophy $(r=0.54,95 \% \mathrm{Cl}: 0.16-0.78, \mathrm{P}<0.001)$.

The above analysis highlighted that FTP annualized change as the stronger factor associated with baseline FTP uptake, potentially because higher baseline FTP uptake was closer in predicting severe FTP change. We further explored whether the longitudinal FTP change could account for the link between baseline FTP cortical uptake and subsequent MRI atrophy. To this end, we modeled a serial mediation assessing different pathways between baseline FTP uptake, longitudinal FTP change and atrophy. We observed from Fig. 4 that the relationship between baseline FTP uptake and longitudinal atrophy was at least partly mediated by the longitudinal FTP cortical change (indirect effect: $0.0107, P=0.04$ ). After mediation, the direct association of baseline FTP uptake with MRI atrophy became nonsignificant $(\mathrm{P}=$ 0.92).

\section{Relationship to cognitive change}

The MMSE and CDRSB assessments were employed to measure clinical cognitive decline. The change of these scores was annualized by (follow-up score - baseline score)/ follow-up time. This analysis denoted that MMSE score changed by $-2.9 \pm-1.2$ scores per year (one-sample t test, $\mathrm{P}<0.001$ ) and CDRSB score increased by $1.25 \pm 1.02$ scores per year (one-sample t test, $P<0.001$ ). Annualized MMSE decrease was moderately correlation with longitudinal FTP increase $(r=-0.34, P<0.01)$, in contrast with poorly correlation with longitudinal atrophy $(r=0.12, P=0.57)$ and baseline FTP cortical uptake $(r=0.13, P=$ 0.55). There were no significant correlations between annualized CDRSB increase with baseline FTP, longitudinal FTP change and atrophy in the cerebral cortex.

\section{Discussion}

In this longitudinal study of tau change and MRI atrophy, we examined the associations between baseline FTP binding level and subsequent neurodegeneration (FTP accumulation and MRI cortical atrophy) over time across $\mathrm{MCl}$ patients. In the $\mathrm{MCl}$ progression, we observed intimated link between FTP accumulation and cortical brain atrophy on the voxelwise analyses, especially in bilateral temporoparietal regions. And longitudinal FTP change has mediated the relationship between baseline FTP uptake and subsequent atrophy across $\mathrm{MCl}$ participants. Finally, we found that longitudinal FTP change, but not baseline FTP uptake or longitudinal cortical atrophy, was significantly correlated with the annualized cognitive change as assessed by MMSE. 
Previous neuroimaging studies examining the association between longitudinal tau change and cortical atrophy in healthy elders and AD patients, and it remained unclear what happened and which association in symptomatic patients with $\mathrm{MCl}$. Our study extended this gap and the main finding was that the spatial topology of FTP accumulation and its annualized change rate $(6-11 \%$ per year) in $\mathrm{MCl}$, which was predominantly middle-inferior temporal cortex. This is in line with previous neuroimaging and pathologic studies in $\mathrm{MCl}$ neurodegeneration showing the similar topology and spread of tau pathology [24, 25]. It was worth noting that the annualized FTP percentage rate for $\mathrm{MCl}$ group in this work was rather than for healthy elders (2-3\% per year) [15]. Considering the sensitivity of FTP and the spread pattern of FTP accumulation may be different in various clinical symptoms (i.e., stable $\mathrm{MCl}$, developing $\mathrm{MCl}$ or reversed $\mathrm{MCl})[26,27]$, it remained to confirmed that the generalizability of our observed rates of longitudinal FTP accumulation in $\mathrm{MCl}$ group. We further used complementary voxelwise and ROI-based analyses to reveal the association between longitudinal FTP change and atrophy. The annualized rate of FTP accumulation and cortical atrophy were considerably stronger and were present across all prior regions. There was a moderate association between the two spatial patterns (Fig. 2 and Fig. 3). Emerging evidence suggested that FTP pathology may present before neurodegeneration starts and may propagate its effects on cognition ability through structural pathology $[28,29]$. Thus, it might be expected that the retention of tau may increase the risk of brain atrophy, in that the higher tau accumulation rate and magnitude, the downstream regions related to severe atrophy. This is consistent with the previous cross-sectional finding that the rate of tau accumulation correlated with atrophy pattern over time $[6,12]$.

Evidence from mediation models had emerged a plausible hypothesis that baseline cortical FTP uptake had a delayed and indirect, longitudinal FTP change-mediated association with longitudinal cortical atrophy. Previous longitudinal MRI data had estimated that baseline tau pathology, rather than amyloid pathology, was a major driver of local neurodegeneration and highlight the relevance of baseline tau uptake level as a precision way to predict individual patient's cortical atrophy [14]. We observed that baseline tau uptake was more closely correlated with longitudinal tau change, beyond the longitudinal cortical atrophy. We also found that those individuals with high rates of tau change had elevated baseline tau uptake and atrophy, rather than the lower patients. These observations expand on previous findings from longitudinal studies by providing evidence that baseline tau level as an important role regarding not only the longitudinal brain atrophy but also longitudinal tau accumulation. Previous clinicopathological studies both PET and cerebrospinal fluid data suggested that synergy tau and atrophy were correlated with brain dysfunction and cognitive deficits $[14,30,31]$. Together, growing evidence suggested that baseline tau uptake may reflect the presence of subsequent tau change, which had a predictive ability to cortical atrophy over time. These findings had potential ramifications for clinical trials in that based-tau measurements might be superior to track pathology trajectories during $\mathrm{MCl}$ progression. Contrary to expectations, we observed that clinical cognitive change as assessed by MMSE and CDRSB was only associated with longitudinal cortical FTP increase. There were no significant correlations between clinical decline and baseline tau uptake, similar in longitudinal cortical atrophy. These weak correlations may be related to the small sample size or intrinsic noise interferences both clinical assessments and imaging [14]. 
A strength of this prospective study was that we used longitudinal FTP PET and structural MRI imaging in individuals across $\mathrm{MCl}$ spectrum. There were some limitations that should be pointed to appropriately interpret our results. Firstly, the small sample size may had limited our statistical power and prevented generalization, and especially to explore the patterns of longitudinal tau change and cortical atrophy (uncorrected $\mathrm{P}$ value). Secondly, the $\mathrm{MCl}$ patients of this study is collected from ADNI database, which may limit the appropriate follow-up visits (only two-time points) and further time points would enable a more detailed characterization of neurodegeneration trajectories. Finally, our cohort only included the progression of $\mathrm{MCl}$ stage and would not be extrapolated to more serve stage (AD or dementia), and also lack amyloid PET to determine the more comprehensive pathological trajectories.

\section{Conclusions}

In summary, in this longitudinal study, we investigated the voxelwise patterns of tau accumulation and cortical atrophy over time and neurodegeneration trajectories in symptomatic patients with $\mathrm{MCl}$. These results suggested that baseline cortical FTP uptake had a delayed and indirect, longitudinal FTP changemediated association with longitudinal cortical atrophy. These findings highlighted the importance of based-tau measurements to track the $\mathrm{MCl}$ degeneration and further halt the pathology progression and delay cognitive deficits.

\section{Abbreviations}

$A D=$ Alzheimer's disease $; A P C=$ annualized percentage change $; C D R S B=$ clinical Dementia Rating sum of boxes; flortaucipir = FTP; $\mathrm{GM}=$ gray matter; $\mathrm{PVE}=$ partial volume effect; $\mathrm{MCl}=$ mild cognitive impairment; $\mathrm{MMSE}=$ mini-mental state examination; $\mathrm{WM}=$ white matter; $\mathrm{SUVR}=$ standardized uptake value ratio; $\mathrm{ROI}=$ region of interest;

\section{Declarations}

\section{Ethics approval and consent to participate}

All procedures performed in studies involving human participants were in accordance with the ethical standards of the institutional and/or national research committee and with the 1964 Helsinki Declaration and its later amendments or comparable ethical standards. All MCl patients signed written consent for evaluation of their data and the institutional review board of the ADNI approved this analysis.

\section{Consent for publication}

Not applicable. 


\section{Availability of data and materials}

Please contact the corresponding author for data requests or download in ADNI database (http://adni.loni.usc.edu/).

\section{Competing interests}

The authors declare that they have no competing interests.

\section{Funding}

This work was supported by grants from the Key Project of Natural Science in Universities in Anhui Province (KJ2018A0111), Open Research Fund of Anhui Key Laboratory of Detection Technology and Energy Saving Devices, Anhui Polytechnic University (2017070503B026-A01).

\section{Authors' contributions}

GX designed the study. GX and SZZ collected the data. ZLZ and XFY developed the experimental model and analyzed the data. JJJ performed clinical assessment and supported the analysis. JJ gave guidance for medical statistics in this study. ZHC, JJJ and GX supervised the study. All authors interpreted data results, drafted, and edited manuscript.

\section{Acknowledgements}

Data collection and sharing for this project was funded by the Alzheimer's Disease Neuroimaging Initiative (ADNI) (National Institutes of Health Grant U01 AG024904) and DOD ADNI (Department of Defense award number W81XWH-12-2-0012). ADNI is funded by the National Institute on Aging, the National Institute of Biomedical Imaging and Bioengineering, and through generous contributions from the following: AbbVie, Alzheimer's Association; Alzheimer's Drug Discovery Foundation; Araclon Biotech; BioClinica, Inc.; Biogen; Bristol-Myers Squibb Company; CereSpir, Inc.; Cogstate; Eisai Inc.; Elan Pharmaceuticals, Inc.; Eli Lilly and Company; Eurolmmun; F. Hoffmann-La Roche Ltd and its affiliated company Genentech, Inc.; Fujirebio; GE Healthcare; IXICO Ltd.; Janssen Alzheimer Immunotherapy Research \& Development, LLC.; Johnson \& Johnson Pharmaceutical Research \& Development LLC.; Lumosity; Lundbeck; Merck \& Co., Inc.; Meso Scale Diagnostics, LLC.; NeuroRx Research; Neurotrack Technologies; Novartis Pharmaceuticals Corporation; Pfizer Inc.; Piramal Imaging; Servier; Takeda Pharmaceutical Company; and Transition Therapeutics. The Canadian Institutes of Health Research is providing funds to support ADNI clinical sites in Canada. Private sector contributions are facilitated by the Foundation for the National Institutes of Health (www.fnih.org). The grantee organization is the 
Northern California Institute for Research and Education, and the study is coordinated by the Alzheimer's Therapeutic Research Institute at the University of Southern California. ADNI data are disseminated by the Laboratory for Neuro Imaging at the University of Southern California.

\section{References}

1. 2019 Alzheimer's disease facts and figures. Alzheimer's \& Dementia. 2019;15(3):321-87.

2. Hyman BT, Phelps CH, Beach TG, Bigio EH, Cairns NJ, Carrillo MC, et al. National Institute on AgingAlzheimer's Association guidelines for the neuropathologic assessment of Alzheimer's disease. Alzheimer's \& dementia : the journal of the Alzheimer's Association. 2012;8(1):1-13.

3. Jagust W. Imaging the evolution and pathophysiology of Alzheimer disease. Nat Rev Neurosci. 2018;19(11):687-700.

4. Giannakopoulos P, Herrmann FR, Bussiere T, Bouras C, Kovari E, Perl DP, et al. Tangle and neuron numbers, but not amyloid load, predict cognitive status in Alzheimer's disease. Neurology. 2003;60(9):1495-500.

5. Nelson PT, Alafuzoff I, Bigio EH, Bouras C, Braak H, Cairns NJ, et al. Correlation of Alzheimer disease neuropathologic changes with cognitive status: a review of the literature. J Neuropathol Exp Neurol. 2012;71(5):362-81.

6. Bejanin A, Schonhaut DR, La Joie R, Kramer JH, Baker SL, Sosa N, et al. Tau pathology and neurodegeneration contribute to cognitive impairment in Alzheimer's disease. Brain. 2017; 140(12):3286-300.

7. Jack CR, Jr., Knopman DS, Jagust WJ, Petersen RC, Weiner MW, Aisen PS, et al. Tracking pathophysiological processes in Alzheimer's disease: an updated hypothetical model of dynamic biomarkers. The Lancet Neurology. 2013;12(2):207-16.

8. Nelson PT, Jicha GA, Schmitt FA, Liu H, Davis DG, Mendiondo MS, et al. Clinicopathologic correlations in a large Alzheimer disease center autopsy cohort: neuritic plaques and neurofibrillary tangles "do count" when staging disease severity. J Neuropathol Exp Neurol. 2007;66(12):1136-46.

9. Cho H, Choi JY, Hwang MS, Lee JH, Kim YJ, Lee HM, et al. Tau PET in Alzheimer disease and mild cognitive impairment. Neurology. 2016;87(4):375-83.

10. Nasrallah IM, Chen YJ, Hsieh MK, Phillips JS, Ternes K, Stockbower GE, et al. (18)F-Flortaucipir PET/MRI Correlations in Nonamnestic and Amnestic Variants of Alzheimer Disease. Journal of nuclear medicine : official publication, Society of Nuclear Medicine. 2018;59(2):299-306.

11. Jack CR, Jr., Wiste HJ, Weigand SD, Therneau TM, Lowe VJ, Knopman DS, et al. Defining imaging biomarker cut points for brain aging and Alzheimer's disease. Alzheimer's \& dementia : the journal of the Alzheimer's Association. 2017;13(3):205-16.

12. Xia C, Makaretz SJ, Caso C, McGinnis S, Gomperts SN, Sepulcre J, et al. Association of In Vivo [18F]AV-1451 Tau PET Imaging Results With Cortical Atrophy and Symptoms in Typical and Atypical Alzheimer Disease. JAMA Neurol. 2017;74(4):427-36. 
13. Hanseeuw BJ, Betensky RA, Jacobs HIL, Schultz AP, Sepulcre J, Becker JA, et al. Association of Amyloid and Tau With Cognition in Preclinical Alzheimer Disease: A Longitudinal Study. JAMA Neurol. 2019.

14. La Joie R, Visani AV, Baker SL, Brown JA, Bourakova V, Cha J, et al. Prospective longitudinal atrophy in Alzheimer's disease correlates with the intensity and topography of baseline tau-PET. Science translational medicine. 2020;12(524).

15. Harrison TM, La Joie R, Maass A, Baker SL, Swinnerton K, Fenton L, et al. Longitudinal tau accumulation and atrophy in aging and alzheimer disease. Ann Neurol. 2019;85(2):229-40.

16. Ashburner J, Ridgway GR. Symmetric diffeomorphic modeling of longitudinal structural MRI. Frontiers in neuroscience. 2012;6:197.

17. Fleisher AS, Joshi AD, Sundell KL, Chen YF, Kollack-Walker S, Lu M, et al. Use of white matter reference regions for detection of change in florbetapir positron emission tomography from completed phase 3 solanezumab trials. Alzheimer's \& dementia : the journal of the Alzheimer's Association. 2017;13(10):1117-24.

18. Southekal S, Devous MD, Sr., Kennedy I, Navitsky M, Lu M, Joshi AD, et al. Flortaucipir F 18 Quantitation Using Parametric Estimation of Reference Signal Intensity. Journal of nuclear medicine : official publication, Society of Nuclear Medicine. 2018;59(6):944-51.

19. Muller-Gartner HW, Links JM, Prince JL, Bryan RN, McVeigh E, Leal JP, et al. Measurement of radiotracer concentration in brain gray matter using positron emission tomography: MRI-based correction for partial volume effects. Journal of cerebral blood flow and metabolism : official journal of the International Society of Cerebral Blood Flow and Metabolism. 1992;12(4):571-83.

20. Gonzalez-Escamilla G, Lange C, Teipel S, Buchert R, Grothe MJ, Alzheimer's Disease Neuroimaging I. PETPVE12: an SPM toolbox for Partial Volume Effects correction in brain PET - Application to amyloid imaging with AV45-PET. Neuroimage. 2017;147:669-77.

21. Xia M, Wang J, He Y. BrainNet Viewer: a network visualization tool for human brain connectomics. PLoS One. 2013;8(7):e68910.

22. Tingley D, Yamamoto T, Hirose K, Keele L, Imai K. mediation:RPackage for Causal Mediation Analysis. Journal of Statistical Software. 2014;59(5):38 \%J Journal of Statistical Software.

23. Jansen WJ, Ossenkoppele R, Knol DL, Tijms BM, Scheltens P, Verhey FR, et al. Prevalence of cerebral amyloid pathology in persons without dementia: a meta-analysis. JAMA. 2015;313(19):1924-38.

24. Hanseeuw BJ, Betensky RA, Schultz AP, Papp KV, Mormino EC, Sepulcre J, et al. Fluorodeoxyglucose metabolism associated with tau-amyloid interaction predicts memory decline. Ann Neurol. 2017;81(4):583-96.

25. Adams JN, Lockhart SN, Li L, Jagust WJ. Relationships Between Tau and Glucose Metabolism Reflect Alzheimer's Disease Pathology in Cognitively Normal Older Adults. Cereb Cortex. 2019;29(5):1997-2009.

26. Josephs KA, Whitwell JL, Tacik P, Duffy JR, Senjem ML, Tosakulwong N, et al. [18F]AV-1451 tau-PET uptake does correlate with quantitatively measured 4R-tau burden in autopsy-confirmed corticobasal 
degeneration. Acta neuropathologica. 2016;132(6):931-3.

27. Smith R, Puschmann A, Scholl M, Ohlsson T, van Swieten J, Honer M, et al. 18F-AV-1451 tau PET imaging correlates strongly with tau neuropathology in MAPT mutation carriers. Brain. 2016;139(Pt 9):2372-9.

28. Spillantini MG, Goedert M. Tau protein pathology in neurodegenerative diseases. Trends in neurosciences. 1998;21(10):428-33.

29. Jack CR, Jr., Holtzman DM. Biomarker modeling of Alzheimer's disease. Neuron. 2013;80(6):1347-58.

30. Wang L, Benzinger TL, Su Y, Christensen J, Friedrichsen K, Aldea P, et al. Evaluation of Tau Imaging in Staging Alzheimer Disease and Revealing Interactions Between $\beta$-Amyloid and Tauopathy. JAMA neurology. 2016;73(9):1070-7.

31. Sepulcre J, Grothe MJ, Sabuncu M, Chhatwal J, Schultz AP, Hanseeuw B, et al. Hierarchical Organization of Tau and Amyloid Deposits in the Cerebral Cortex. JAMA neurology. 2017;74(7):81320.

\section{Figures}

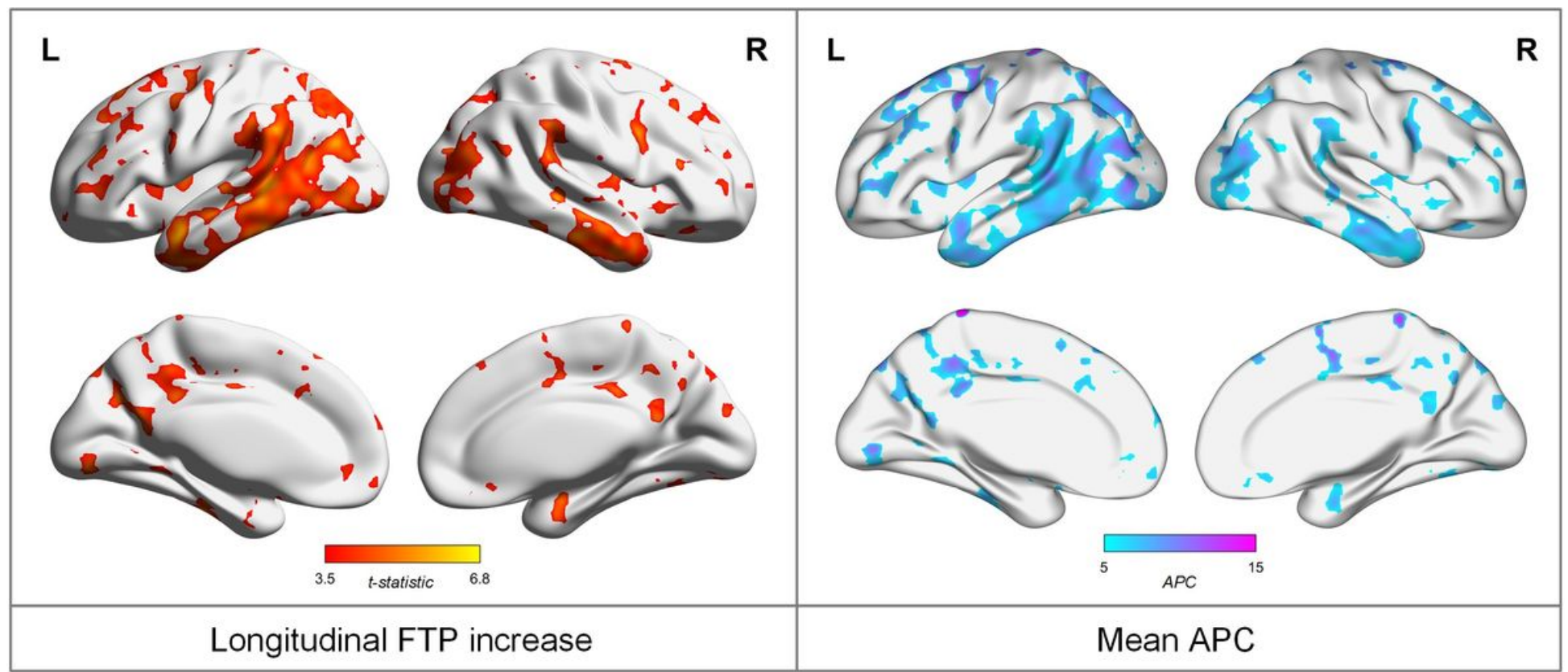

\section{Figure 1}

The longitudinal FTP increase in $\mathrm{MCl}$ patients. The left panel was the result's region of one-sample t test where $\mathrm{MCl}$ patients showed a significant FTP increase over time (voxel $\mathrm{P}<0.001$, uncorrected). The right panel showed that voxelwise average annualized percentage change (APC) in significant FTP change region. 


\section{Longitudinal FTP increase}

A
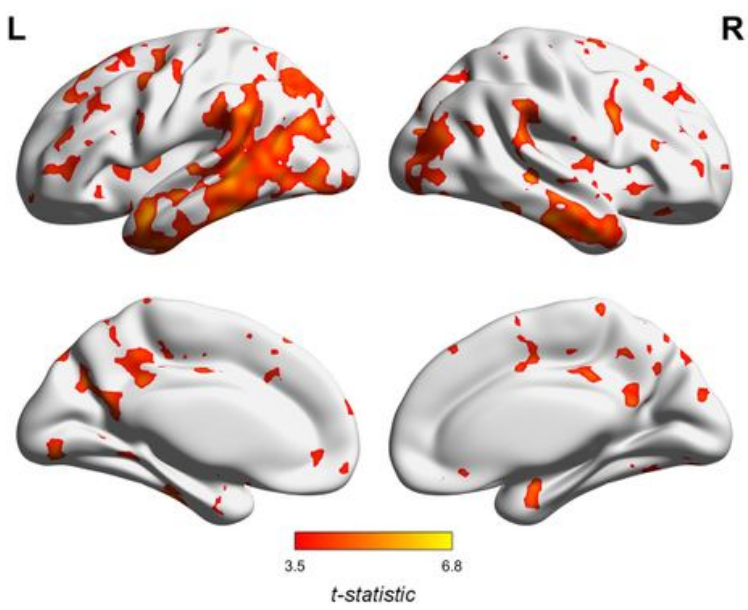

B
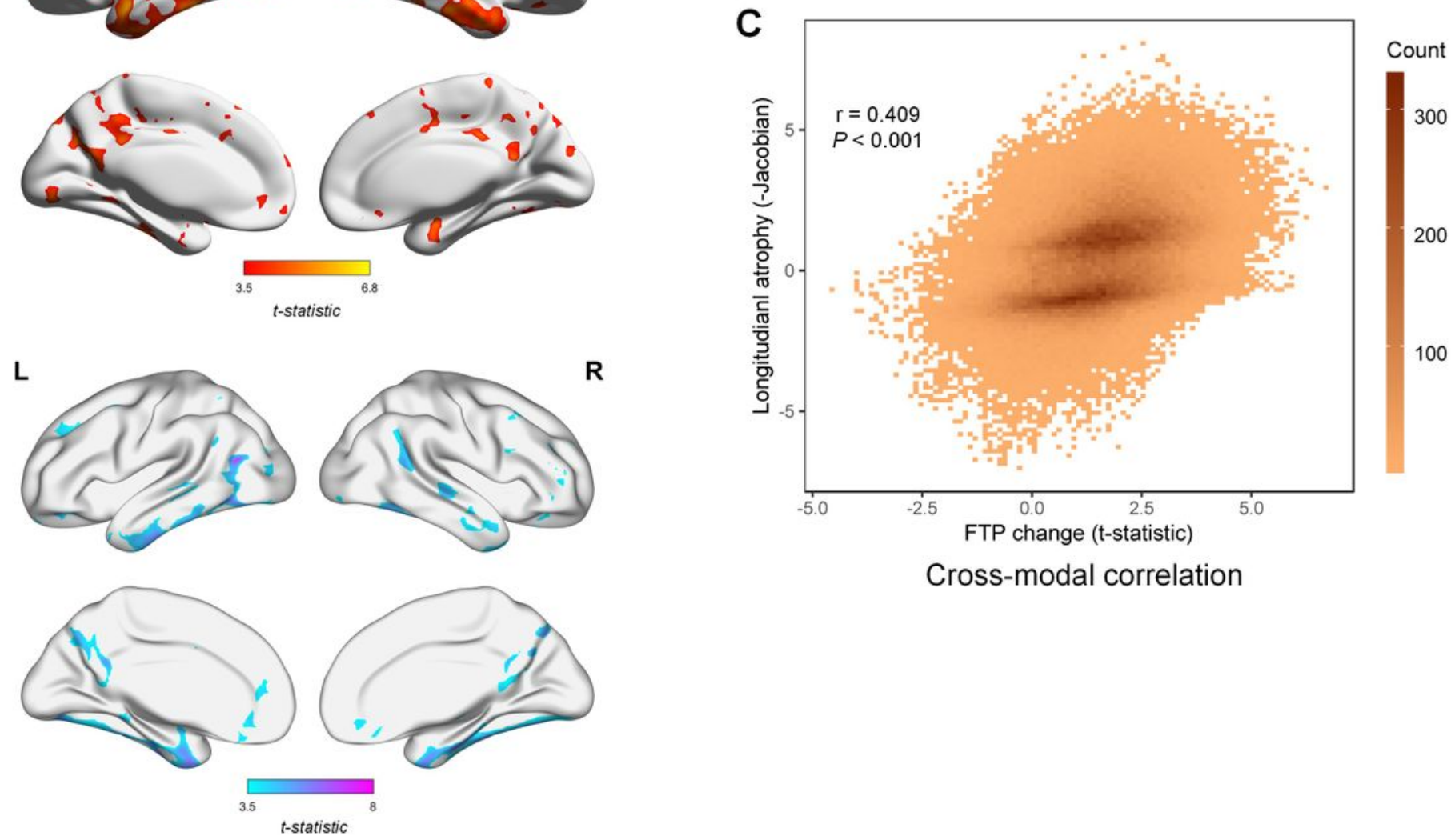

Cross-modal correlation

Longitudinal MRI atrophy

\section{Figure 2}

The spatial statistic maps of FTP accumulation and MRI atrophy and cross-modal correlation. (A) The significant FTP change statistic map derived from FTP change images of each $\mathrm{MCl}$ patient $(n=23$, onesample t test, $P<0.001)$. (B) The significant MRI atrophy statistic map derived from reversed Jacobian rate map of each patient $(n=23$, one-sample t test, $P<0.001)$. (C) The cross-modal correlation between FTP accumulation and MRI atrophy derived from voxelwise statistic maps (T value). Correlation was assessed on all voxels of the cerebral cortex.
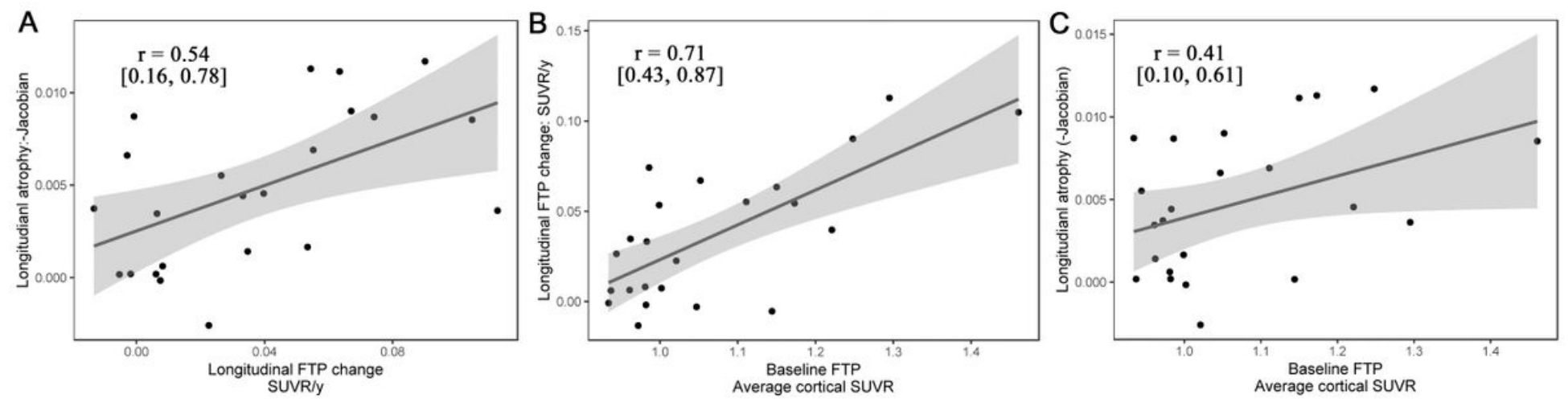
Figure 3

Bivariate associations between baseline FTP and subsequent longitudinal FTP change/MRI atrophy across the $23 \mathrm{MCl}$ patients. For $95 \%$ confidence interval $(95 \% \mathrm{Cl})$, the bootstrapping with 1000 permutations was performed.

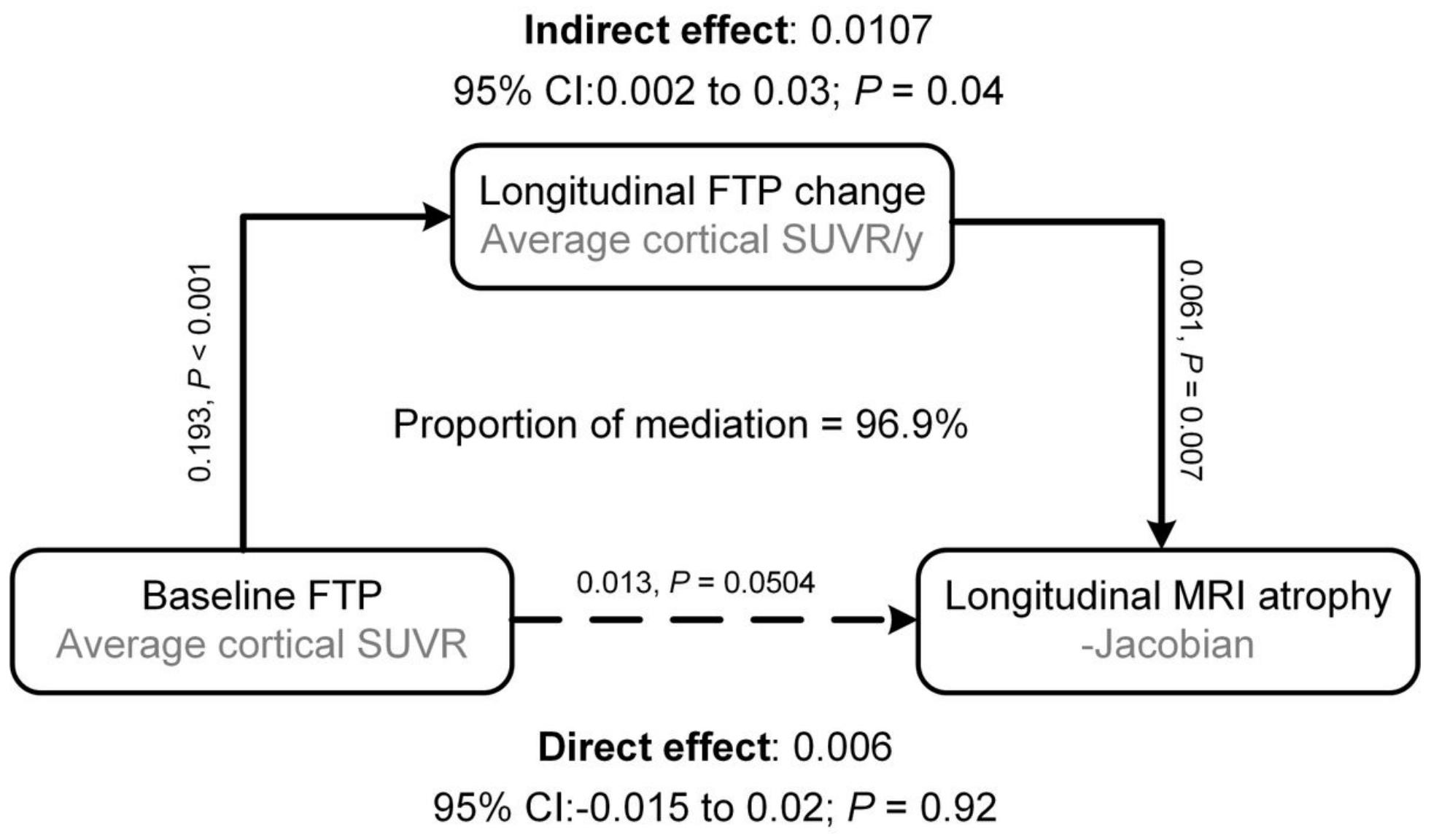

Figure 4

Diagram of mediation model pathway relating baseline FTP, longitudinal FTP change and MRI atrophy. Altogether, the pathways explain $96.9 \%$ of the direct effect. Mediation analysis showed that longitudinal FTP change mediated the effect of baseline FTP on longitudinal MRI atrophy.

\section{Supplementary Files}

This is a list of supplementary files associated with this preprint. Click to download.

- appendix.docx 